\title{
Erratum zu: Energie-Autarkie und Energie-Autonomie in Theorie und Praxis
}

\author{
Jürgen Deuschle $\cdot$ Wolfgang Hauser - Marco Sonnberger · \\ Jan Tomaschek · Lukasz Brodecki · Ulrich Fahl
}

Online publiziert: 4. November 2015

(C) Springer Fachmedien Wiesbaden 2015

Erratum to: Z Energiewirtsch (2015) 39:151-162

DOI 10.1007/s12398-015-0160-5

Due to a typesetting error the name of one co-author was incorrect. Please find the correct name below:

\section{Jürgen Deuschle}

Die Online-Version des Originalartikels ist unter doi:10.1007/s12398-015-0166-z zu finden.

Dr. J. Tomaschek $(\bowtie) \cdot$ L. Brodecki $\cdot$ Dr. U. Fahl Institut für Energiewirtschaft und Rationelle Energieanwendung (IER), Universität Stuttgart,

Stuttgart, Deutschland

E-Mail: jo@ier.uni-stuttgart.de

J. Deuschle $\cdot$ Dr. W. Hauser $\cdot$ M. Sonnberger

Zentrum für interdisziplinäre Risiko- und Innovationsforschung,

Universität Stuttgart (ZIRIUS),

Stuttgart, Deutschland 